\title{
On Maps with Given Jacobians involving the Heat Equation
}

\author{
Albert Avinyó $^{1} \quad$ Joan Solà-Morales ${ }^{2} \quad$ Marta València ${ }^{2}$
}

\begin{abstract}
In this paper we present and analyze two new algorithms to construct a smooth diffeomorphism of a domain with prescribed jacobian function. The first one is free from any restriction on the boundary, while the second one produces a diffeomorphism that coincides with the identity map on the boundary of the domain. Both are based on the solution of an initial value problem for the linear heat equation, and the second also uses solutions of the Stokes system of Fluid Mechanics.
\end{abstract}

Mathematics Subject Classification (1991). 35J60, 35B65.

Keywords. Prescribed jacobian equation, Hölder regularity, heat equation, Stokes system.

\section{Introduction}

Let $\Omega \subset \mathbb{R}^{n}$ be a bounded domain and $f: \bar{\Omega} \rightarrow \mathbb{R}^{+}$a function satisfying $\inf _{\bar{\Omega}} f>0$ and $\int_{\Omega} f=|\Omega|$. The problem we are dealing with is to construct a smooth diffeomorphism $\Phi: \bar{\Omega} \rightarrow \bar{\Omega}$ such that

$$
\operatorname{det}(D \Phi)(x)=f(x), \quad x \in \Omega .
$$

This problem arose during the modelling of the construction of the geographical representations called area-cartograms (see, for example, Dorling [8]). In this case $f$ should be a piecewise constant function that represents some characteristic quantity, such as the number of inhabitants or the gross domestic product of each region. Nevertheless, the results of this paper will deal only with more regular functions. The prescribed jacobian equation (1) has also applications in several problems of Mathematical Physics, such as the problem of equilibrium of gases considered in Section A.2.3 of the book of Dacorogna [6]. It can also be used to generate random distributions of particles on a domain with a given probability density (see Russo [20]).

\footnotetext{
${ }^{1}$ Partially supported by the projects PB95-0629-C02-02 of the DGICYT and PB98-0932C02-01 of CICYT, Spain.
} 
For a given $f$ and under reasonable hypotheses, it is quite clear that equation (1) will have at least one solution, but it is also clear that the solutions will not be at all unique. This has been one of the main motivations of our work: to obtain a unique $\Phi$ in terms of $f, \Omega$ and a boundary condition, should there be one, by means of a definite algorithm or a formula. This algorithm should be free from arbitrary choices, easy to construct, and produce maps $\Phi$ which are as smooth as possible. We trust that the two algorithms we present, based on the linear heat equation, will represent a nontrivial positive contribution in each one of these three directions.

A natural way of avoiding the non-uniqueness is to look among all the possible solutions for the one that minimizes a suitable cost functional. This gives rise to the Monge-Kantorovich mass transfer problem (see, for example, Brenier [2]) where equation (1) is considered without any additional boundary condition. However, using Brenier's construction, the regularity of $\Phi$, and even its continuity, becomes unclear for nonconvex domains (see Caffarelli [4]), even for some smooth data.

Another approach to solving equation (1), according to Moser [18], which we will follow in this paper, can be interpreted as building the diffeomorphism $\Phi$ by solving a flow problem in the following way: consider a conservation law of the type

$$
\rho_{t}+\operatorname{div}(\rho F)=0
$$

for $0<t<T$ and subject to the initial and final conditions

$$
\rho(0, x)=f(x) \quad \text { and } \quad \rho(T, x)=1 .
$$

This equation describes the time evolution of a density $\rho(t, x)$ transported by a velocity field $F(t, x)$ from the original density $\rho(0, x)=f(x)$ to the homogeneous density $\rho(T, x)=1$. So, if we take $x(t)$ as the solution of the initial value problem

$$
\left\{\begin{aligned}
x^{\prime}(t) & =F(t, x(t)), \\
x(0) & =x_{0},
\end{aligned}\right.
$$

then, at least formally, it follows that the map $\Phi\left(x_{0}\right)=x(T)$ satisfies (1). In this situation, the domain $\Omega$ will remain invariant by $\Phi$ if we ask $F(t, x)$ also to be parallel to $\partial \Omega$ and, if one wishes $\Phi$ keeps the points of the boundary of this domain fixed, it is necessary for $F(t, x)$ to vanish for all $x \in \partial \Omega$.

Moser's construction in [18] corresponds to taking $\rho=t+(1-t) f$ and $F=\nabla u /(t+(1-t) f)$ with $T=1$ (in this case the conservation law (2) becomes the Poisson equation) when he proved the existence of a $\Phi \in \mathcal{C}^{\infty}$ that solves (1) if $f$ is a $\mathcal{C}^{\infty}$ function. Dacorogna and Moser [7] and Rivière and Ye [19] (see also the references therein) have given extensions of this result in order to obtain the existence at least of a solution of (1) with keeping the points of the boundary fixed and having as much regularity as possible for a given $f$ belonging to Hölder or Sobolev spaces. Recently, Burago and Kleiner [3] and McMullen [16] have 
found an example of a continuous function $f$ such that there is no Lipschitz function $\Phi$ that satisfies (1) in a weak form.

In the framework of the Monge-Kantorovich mass transfer problem, Evans and Gangbo [9] have used this differential-equations-based approach with $F=$ $|\nabla u|^{p-2} \nabla u$ where $u$ satisfies $-\operatorname{div}\left(|\nabla u|^{p-2} \nabla u\right)=f-1$, and then taking $p \rightarrow \infty$.

Following Moser's idea, in this paper we present two algorithms to solve equation (1), the first one without any boundary condition and the second one imposing that $\Phi(x)=x$, for all $x \in \partial \Omega$.

To obtain the first algorithm, we take $\rho=u$ and $F=-\nabla u / u$, with $F$ parallel to the boundary of the domain $\Omega$. With this choice, conservation law (2) is transformed into the heat equation with homogeneous Neumann boundary conditions. We observe, in this case, that the initial data $u(0, x)=f(x)$ tend to their spatial average when $t \rightarrow \infty$, and so, at least formally, for $T=\infty$, condition (3) is satisfied. In this way, the solution of equation (1) obtained is easy to construct and, due to the smoothing properties of the heat equation, will have the expected regularity. Moreover, it has the same isotropy property as the heat equation. Thus, one can hope that this solution could be a suitable algorithm with the properties mentioned above.

We remark that this algorithm is not completely new. A variation of it, which corresponds to the case $\Omega=\mathbb{R}^{n}$, was used by Russo (see Section 5 of [20]) to generate distributions of particles in the space according to a given probability $f$.

The second algorithm is a variation of the first one, but the points of $\partial \Omega$ are kept fixed. In this case, we have to modify the previous $F$ to a new $\bar{F}$ so that it vanishes at $\partial \Omega$ but keeping $\operatorname{div} u F=\operatorname{div} u \bar{F}$. This can be done in many ways, for example by using suitable, but arbitrary, cut-off functions, as in some references above. However, in this paper, we will use the classical tool of the Stokes system of hydrodynamics which enables us to maintain the isotropy and to prove the same regularity results as in the previous algorithm. This modification can be interpreted as simply adding viscosity to the transport flow.

The rest of this paper is organized as follows. In Section 1 we state Theorem 1 and Theorem 2, which are the main results of the paper. Theorem 1 is concerned with the first algorithm and Theorem 2 with the second. We also present some pictures as examples of a numerical implementation of these algorithms. In Section 2 we state and prove a preliminary result, Theorem 3, concerning existence, uniqueness, and regularity of the solution to certain types of ordinary differential equations which we use in the proofs of Theorem 1 and Theorem 2 . Finally, Section 3 and Section 4 are devoted to the proofs of each of these two theorems, respectively.

The authors are grateful to X. Cabré, V.I. Solonnikov and D. Ye for several useful references, and to J. Grané and J. Masdemont for their help in the numerical calculations. 


\section{Main results}

The following two theorems present the two algorithms considered and show that each one really defines a unique smooth diffeomorphism under our regularity requirements for the data. We point out that they achieve the optimal regularity, at least in some Hölder spaces, in the sense that, if we take the jacobian function $f$ to be of class $\mathcal{C}^{0, \alpha}$, we obtain a diffeomorphism $\Phi$ of class $\mathcal{C}^{1, \alpha}$.

Theorem 1 Let $\Omega \subset \mathbb{R}^{n}$ be a bounded domain of class $\mathcal{C}^{2, \alpha^{\prime}}, 0<\alpha^{\prime}<1$, and $f: \bar{\Omega} \rightarrow \mathbb{R}^{+}$a function belonging to the space $\mathcal{C}^{0, \alpha}(\bar{\Omega})$ for some $0<\alpha<\alpha^{\prime}$, $\inf _{\bar{\Omega}} f>0$ and satisfying $\int_{\Omega} f=|\Omega|$. Let $u(t, x)$ be the solution to the initial value problem for the linear heat equation with homogeneous Neumann boundary conditions

$$
\left\{\begin{aligned}
u_{t}(t, x) & =\Delta u(t, x), & & x \in \Omega, \quad t>0, \\
u(0, x) & =f(x), & & x \in \Omega, \\
u_{\nu}(t, x) & =0, & & x \in \partial \Omega, \quad t>0,
\end{aligned}\right.
$$

where $\nu$ denotes the outer unit normal vector to the boundary of $\Omega$. Then,

a) For all $x_{0} \in \bar{\Omega}$, the initial value problem for the ordinary differential equation

$$
\left\{\begin{aligned}
x^{\prime}(t) & =\frac{-\nabla u(t, x(t))}{u(t, x(t))}, \quad t>0, \\
\lim _{t \rightarrow 0^{+}} x(t) & =x_{0}
\end{aligned}\right.
$$

has a unique solution $x(t) \in \mathcal{C}^{1}((0, \infty), \bar{\Omega})$, and $\lim _{t \rightarrow \infty} x(t)$ exists and belongs to $\bar{\Omega}$.

b) The map $\Phi: \bar{\Omega} \rightarrow \bar{\Omega}$ defined by $\Phi\left(x_{0}\right)=\lim _{t \rightarrow \infty} x(t)$ is a diffeomorphism of $\bar{\Omega}$ such that $\Phi, \Phi^{-1} \in \mathcal{C}^{1, \alpha}(\bar{\Omega})$, and it satisfies that

$$
\operatorname{det}(D \Phi)(x)=f(x), \quad x \in \bar{\Omega} .
$$

Theorem 2 Let $\Omega, f(x)$ and $u(t, x)$ be as in Theorem 1, and for each $t>0$, let $V(t, x)$ be the solution, in a weak sense, to the following Stokes problem,

$$
\left\{\begin{aligned}
\Delta V(t, x) & =\nabla p(t, x), & & x \in \Omega, \\
\operatorname{div} V(t, x) & =0, & & x \in \Omega, \\
V(t, x) & =\nabla u(t, x), & & x \in \partial \Omega .
\end{aligned}\right.
$$

Then,

a) For all $x_{0} \in \bar{\Omega}$, the initial value problem for the ordinary differential equation

$$
\left\{\begin{aligned}
x^{\prime}(t) & =-\frac{\nabla u(t, x(t))-V(t, x(t))}{u(t, x(t))}, \quad t>0, \\
\lim _{t \rightarrow 0^{+}} x(t) & =x_{0},
\end{aligned}\right.
$$


has a unique solution $x(t) \in \mathcal{C}^{1}((0, \infty), \bar{\Omega})$, and $\lim _{t \rightarrow \infty} x(t)$ exists and belongs to $\bar{\Omega}$.

b) The map $\Phi: \bar{\Omega} \rightarrow \bar{\Omega}$ defined by $\Phi\left(x_{0}\right)=\lim _{t \rightarrow \infty} x(t)$ is a diffeomorphism of $\bar{\Omega}$ such that $\Phi, \Phi^{-1} \in \mathcal{C}^{1, \alpha}(\bar{\Omega})$, and it satisfies that

$$
\left\{\begin{aligned}
\operatorname{det}(D \Phi)(x) & =f(x), & & x \in \bar{\Omega}, \\
\Phi(x) & =x, & & x \in \partial \Omega .
\end{aligned}\right.
$$

We remark that the initial value problems (4) and (5) above are singular in the sense that the right hand sides $-\nabla u / u$ or $-(\nabla u-V) / u$ do not have a limit as $t \rightarrow 0$ for most functions $f \in \mathcal{C}^{\alpha}$. Moreover, another difficulty is that we are interested in the value of the solutions for $t=\infty$. Both problems will be solved in Theorem 3 of Section 2.

Next, in Figure 1 we present two series of pictures with numerical computations carried out with the algorithms described in Theorem 1 and Theorem 2, respectively. Here, we take the domain $\Omega$ as the unit disc in the plane and the jacobian function $f$ is defined by

$$
f(x, y)= \begin{cases}1+\varepsilon, & \text { if } y>0 \\ 1-\varepsilon, & \text { if } y<0\end{cases}
$$

for $\varepsilon=0.25,0.5$ and 0.75 . To represent the action of the transformation maps, we have depicted, in the first place, the image of a square grid in the original variables.

Even though this function $f$ does not satisfy the regularity hypotheses of Theorem 1 and Theorem 2, its clear intuitive meaning renders it suitable. Furthermore, this example suggests that our methods are robust enough to work in this discontinuous case too. 


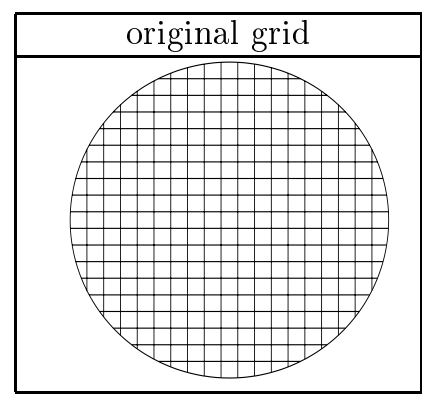

\begin{tabular}{|l|l|l|}
\hline$\epsilon=0.25$ & first algorithm & second algorithm \\
\hline & & \\
\hline & & \\
\hline & & \\
\hline & & \\
\hline
\end{tabular}

Figure 1 


\section{The singular initial and final value problems.}

This section contains a preliminary theorem about the existence, uniqueness and regularity of solutions to certain types of ordinary differential equations. This result is not quoted in the most general form, but in a way appropriate to prove Theorem 1 and Theorem 2. For instance, the existence and uniqueness of solutions could be proved for functions of class $\mathcal{C}^{0,1}$ instead of $\mathcal{C}^{1,0}$.

Theorem 3 Let $\Omega \subset \mathbb{R}^{n}$ be a bounded domain of class $\mathcal{C}^{2,0}$, and $F:(0, \infty) \rightarrow$ $\mathcal{C}^{1,0}\left(\bar{\Omega}, \mathbb{R}^{n}\right)$ be a continuous function such that

$$
\|F(t, \cdot)\|_{\mathcal{C}^{1,0}} \in L^{1}(0, \infty)
$$

and

$$
F(t, x) \cdot \nu(x)=0, \quad \text { for all } x \in \partial \Omega
$$

where $\nu(x)$ denotes the outer unit normal to the boundary of $\Omega$ at $x$. Then,

a) For all $x_{0} \in \bar{\Omega}$, the initial value problem

$$
\left\{\begin{aligned}
x^{\prime}(t) & =F(t, x(t)), \quad t>0 \\
\lim _{t \rightarrow 0^{+}} x(t) & =x_{0}
\end{aligned}\right.
$$

has a unique solution $x_{i n}\left(t ; x_{0}\right) \in \mathcal{C}^{1}((0, \infty), \bar{\Omega})$. Moreover, $\lim _{t \rightarrow \infty} x_{i n}\left(t ; x_{0}\right)$ exists and belongs to $\bar{\Omega}$.

b) For all $x_{\infty} \in \bar{\Omega}$, the final value problem

$$
\left\{\begin{aligned}
x^{\prime}(t) & =F(t, x(t)), \quad t>0 \\
\lim _{t \rightarrow \infty} x(t) & =x_{\infty}
\end{aligned}\right.
$$

has a unique solution $x_{f i}\left(t ; x_{\infty}\right) \in \mathcal{C}^{1}((0, \infty), \bar{\Omega})$. Moreover, $\lim _{t \rightarrow 0^{+}} x_{f i}\left(t ; x_{\infty}\right)$ exists and belongs to $\bar{\Omega}$.

c) The maps $\Phi, \Psi: \bar{\Omega} \rightarrow \bar{\Omega}$ defined by

$$
\Phi\left(x_{0}\right)=\lim _{t \rightarrow \infty} x_{i n}\left(t ; x_{0}\right), \quad \Psi\left(x_{\infty}\right)=\lim _{t \rightarrow 0^{+}} x_{f i}\left(t ; x_{\infty}\right),
$$

satisfy $\Phi, \Psi \in \mathcal{C}^{1,0}(\bar{\Omega}), \Psi=\Phi^{-1}$ and

$$
\operatorname{det}(D \Phi)\left(x_{0}\right)=\exp \left(\int_{0}^{\infty} \operatorname{div} F\left(t, x_{i n}\left(t ; x_{0}\right)\right) d t\right), \quad \text { for all } x_{0} \in \bar{\Omega} .
$$

d) Moreover, if for some $0<\alpha<1$ there exists a constant $C>0$ such that for all $x, y \in \mathcal{C}^{0}((0, \infty), \bar{\Omega})$,

$$
\int_{0}^{\infty}\left|D_{x} F(s, x(s))-D_{x} F(s, y(s))\right| d s \leq C\|x-y\|_{0}^{\alpha},
$$

then $\Phi, \Psi \in \mathcal{C}^{1, \alpha}(\bar{\Omega})$. (Here, $\|\cdot\|_{0}$ denotes the sup-norm). 
Proof: a) First of all, we can construct a bounded linear operator of extension

$$
\mathcal{E}: \mathcal{C}^{1,0}\left(\bar{\Omega}, \mathbb{R}^{n}\right) \rightarrow \mathcal{C}^{1,0}\left(\mathbb{R}^{n}, \mathbb{R}^{n}\right),
$$

such that $(\mathcal{E} F)(x)=F(x)$ if $x \in \bar{\Omega}$ and $\|\mathcal{E} F\|_{\mathcal{C}^{1,0}\left(\mathbb{R}^{n}, \mathbb{R}^{n}\right)} \leq C_{1}\|F\|_{\mathcal{C}^{1,0}\left(\bar{\Omega}, \mathbb{R}^{n}\right)}$ (see, for instance, Appendix 2 of Chapter 6 of Gilbarg and Trudinger [12]). So, instead of the time-dependent vector field $F$, we consider the extended vector field $\mathcal{E} F$, which from now on will be denoted by $\tilde{F}$. We define also $\rho(t):=\|\tilde{F}(t, \cdot)\|_{\mathcal{C}^{1,0}}$, and then $\rho(t) \in L^{1}(0, \infty)$.

Let $T: \mathcal{C}^{0}\left((0, \infty), \mathbb{R}^{n}\right) \times \mathbb{R}^{n} \rightarrow \mathcal{C}^{0}\left((0, \infty), \mathbb{R}^{n}\right)$ be the map

$$
T\left(x(\cdot), x_{0}\right)(t)=x_{0}+\int_{0}^{t} \tilde{F}(s, x(s)) d s .
$$

Using the norm,

$$
\|x\|_{0}^{\prime}=\sup _{t>0}\left\{|x(t)| \exp \left(-2 \int_{0}^{t} \rho(s) d s\right)\right\},
$$

equivalent to the usual sup-norm, we can prove that $T$ is well defined and it satisfies

$$
\begin{aligned}
\left|T\left(x(\cdot), x_{0}\right)(t)-T\left(y(\cdot), x_{0}\right)(t)\right| & \leq \int_{0}^{t} \rho(s)|x(s)-y(s)| d s \\
& \leq\|x-y\|_{0}^{\prime} \int_{0}^{t} \rho(s) \exp \left(2 \int_{0}^{s} \rho(u) d u\right) d s \\
& \leq \frac{1}{2}\|x-y\|_{0}^{\prime}\left(\exp \left(2 \int_{0}^{t} \rho(s) d s\right)-1\right)
\end{aligned}
$$

for all $t>0$. Therefore,

$$
\left\|T\left(x, x_{0}\right)-T\left(y, x_{0}\right)\right\|_{0}^{\prime} \leq \frac{1}{2}\|x-y\|_{0}^{\prime} .
$$

Then, by the Contraction Mapping Principle, it turns out that, for all $x_{0} \in$ $\mathbb{R}^{n}$, there exists a unique fixed point of $T$ which is the solution $x_{i n}\left(t ; x_{0}\right) \in$ $\mathcal{C}^{1}\left((0, \infty), \mathbb{R}^{n}\right)$ of $(6)$.

Moreover, $\lim _{t \rightarrow \infty} T\left(x(t), x_{0}\right)$ always exists since for $0<t_{1}<t_{2}$,

$$
\left|T\left(x(\cdot), x_{0}\right)\left(t_{1}\right)-T\left(x(\cdot), x_{0}\right)\left(t_{2}\right)\right| \leq \int_{t_{1}}^{t_{2}}\|\tilde{F}(s, \cdot)\|_{\mathcal{C}^{0,0}} d s \leq \int_{t_{1}}^{\infty} \rho(s) d s,
$$

and so it can be made as small as one wishes if $t_{1}$ is large enough.

In order to finish the proof of paragraph $a$ ), it remains to show that if $x_{0} \in \bar{\Omega}$ then $x_{i n}\left(t ; x_{0}\right)$ and $\lim _{t \rightarrow \infty} x_{i n}\left(t ; x_{0}\right)$ belong to $\bar{\Omega}$, that is, $\bar{\Omega}$ is invariant by (6).

Since $F(t, x)$ is parallel to $\partial \Omega$ and $\Omega$ is a domain of class $\mathcal{C}^{2,0}$, one can prove that if there exists $t_{1}>0$ such that $x_{i n}\left(t_{1} ; x_{0}\right) \in \partial \Omega$ then $x_{i n}\left(t ; x_{0}\right) \in \partial \Omega$, for 
all $t \in\left(t_{1}-\varepsilon, t_{1}+\varepsilon\right)$. So, on the one hand, if $x_{0} \in \Omega$, by uniqueness of solutions, we have that $x_{i n}\left(t ; x_{0}\right) \in \bar{\Omega}$. On the other hand, if $x_{0} \in \partial \Omega$ one can consider a sequence $\left(x_{0}^{n}\right) \rightarrow x_{0}, x_{0}^{n} \in \Omega$, and then, by the continuity of the fixed point with respect to parameters, we have also $x_{i n}\left(t ; x_{0}\right) \in \bar{\Omega}$.

b) Certainly, similar arguments can be reproduced to obtain the existence and uniqueness of the solution $x_{f i}\left(t ; x_{\infty}\right)$ to the final value problem (7). In this situation, we have to prove that the transformation $S$ defined by

$$
S\left(x(\cdot), x_{\infty}\right)(t)=x_{\infty}-\int_{t}^{\infty} \tilde{F}(s, x(s)) d s,
$$

is a contraction when the space $\mathcal{C}^{0}\left((0, \infty), \mathbb{R}^{n}\right)$ is endowed with the following norm, equivalent to the usual sup-norm,

$$
\|x\|_{0}^{\prime \prime}=\sup _{t>0}\left\{|x(t)| \exp \left(-2 \int_{t}^{\infty} \rho(s) d s\right)\right\} .
$$

c) Let $g: \mathbb{R}^{n} \rightarrow \mathcal{C}^{0}\left((0, \infty), \mathbb{R}^{n}\right)$ be defined by $g\left(x_{0}\right)=x_{i n}\left(t ; x_{0}\right)$, where $x_{i n}\left(t ; x_{0}\right)$ is the fixed point of the operator $T$ defined in (11), for all $x_{0} \in \mathbb{R}^{n}$. If we prove that $T$ is $\mathcal{C}^{1,0}$ then, by the Uniform Contraction Principle (see Theorem 2.2 of Chow and Hale [5]), we will obtain that $g$ belongs to $\mathcal{C}^{1,0}\left(\mathbb{R}^{n}\right)$, and, therefore, the map

$$
\Phi\left(x_{0}\right)=\lim _{t \rightarrow \infty} g_{\mid \bar{\Omega}}\left(x_{0}\right)
$$

will also be of class $\mathcal{C}^{1,0}(\bar{\Omega})$. Obviously, the same will be true for $\Psi=\Phi^{-1}$.

So, we start by proving that $T$ is differentiable (in the sense of Fréchet), that is to say:

$\frac{\left\|T\left(x+h, x_{0}+h_{0}\right)-T\left(x, x_{0}\right)-D T\left(x, x_{0}\right)\left(h, h_{0}\right)\right\|_{0}}{\left\|\left(h, h_{0}\right)\right\|_{0}} \rightarrow 0 \quad$ as $\quad\left\|\left(h, h_{0}\right)\right\|_{0} \rightarrow 0$,

where

$$
D T\left(x(\cdot), x_{0}\right)\left(h(\cdot), h_{0}\right)(t)=h_{0}+\int_{0}^{t} D_{x} \tilde{F}(s, x(s)) h(s) d s,
$$

for all $\left(h, h_{0}\right) \in \mathcal{C}^{0}\left((0, \infty), \mathbb{R}^{n}\right) \times \mathbb{R}^{n}$.

Since $\tilde{F} \in \mathcal{C}^{1,0}\left(\mathbb{R}^{n}, \mathbb{R}^{n}\right)$, we have

$$
\begin{aligned}
& \left|T\left(x(\cdot)+h(\cdot), x_{0}+h_{0}\right)(t)-T\left(x(\cdot), x_{0}\right)(t)-D T\left(x(\cdot), x_{0}\right)\left(h(\cdot), h_{0}\right)(t)\right| \\
= & \left|\int_{0}^{t}\left(\int_{0}^{1}\left(D_{x} \tilde{F}(s, x(s)+\tau h(s))-D_{x} \tilde{F}(s, x(s))\right) h(s) d \tau\right) d s\right| \\
\leq & \left\|\left(h, h_{0}\right)\right\|_{0} \int_{0}^{1}\left(\int_{0}^{\infty}\left|D_{x} \tilde{F}(s, x(s)+\tau h(s))-D_{x} \tilde{F}(s, x(s))\right| d s\right) d \tau .
\end{aligned}
$$


Then, taking $t_{1}>0$ sufficiently small and $t_{2}>t_{1}$ sufficiently large, in order to decompose in a suitable way the integral from 0 to $\infty$, and using that $\|\tilde{F}(t, \cdot)\|_{\mathcal{C}^{1,0}} \in L^{1}(0, \infty)$ and $D_{x} \tilde{F}$ is uniformly continuous in $\left[t_{1}, t_{2}\right]$, it is easy to obtain that $T$ is Fréchet differentiable.

Moreover, since we have,

$$
\begin{aligned}
& \sup _{\left\|\left(h, h_{0}\right)\right\|_{0} \leq 1}\left\|\left(D T\left(x+y, x_{0}+y_{0}\right)-D T\left(x, x_{0}\right)\right)\left(h, h_{0}\right)\right\|_{0} \\
\leq & \int_{0}^{\infty}\left|D_{x} \tilde{F}(s, x(s)+y(s))-D_{x} \tilde{F}(s, x(s))\right| d s,
\end{aligned}
$$

the same decomposition of the integral can be used to see that $D T$ is $\mathcal{C}^{0}$.

To finish the proof of paragraph $c$ ), we must evaluate the jacobian of the map $\Phi$. To do this, let us approximate the previous initial value problem (6) by the sequence of regular initial value problems:

$$
\left\{\begin{aligned}
x_{n}^{\prime}(t) & =F\left(t, x_{n}(t)\right), \quad 1 / n \leq t \leq n, \\
x_{n}(1 / n) & =x_{0}^{n},
\end{aligned}\right.
$$

where $x_{0}^{n}:=x_{i n}\left(1 / n ; x_{0}\right)$, for all $n \in \mathbb{N}$ and we define $\Phi_{n}: \bar{\Omega} \rightarrow \bar{\Omega}$ by $\Phi_{n}\left(x_{0}\right)=$ $x_{n}\left(n ; x_{0}^{n}\right)$. We leave to the reader to show that $\Phi_{n}$ tends to $\Phi$ as $n \rightarrow \infty$ in the $\mathcal{C}^{1,0}(\bar{\Omega})$-norm. Obviously, all properties proved for $\Phi$ before are also true for $\Phi_{n}$. Moreover,

$$
\begin{aligned}
\operatorname{det} D \Phi_{n}\left(x_{0}^{n}\right) & =\exp \left(\int_{1 / n}^{n} \operatorname{div} F\left(t, x_{n}\left(t ; x_{0}^{n}\right)\right) d t\right) \\
& =\exp \left(\int_{1 / n}^{n} \operatorname{div} F\left(t, x_{i n}\left(t ; x_{0}\right)\right) d t\right)
\end{aligned}
$$

(see Corollary 3.1 of Hartman [13]). Then, since $\Phi_{n}$ tends to $\Phi$ as $n \rightarrow \infty$ in the $\mathcal{C}^{1,0}(\bar{\Omega})$-norm, by passing to the limit, we obtain the inequality (8) as was claimed.

d) Following the notation of paragraph $c$ ), we have to prove that $g_{\mid \bar{\Omega}}\left(x_{0}\right)=$ $x_{i n}\left(t ; x_{0}\right)$ belongs to $\mathcal{C}^{1, \alpha}(\bar{\Omega})$. From paragraph $\left.c\right)$, we know that $g \in \mathcal{C}^{1,0}(\bar{\Omega})$ and

$$
D g_{\mid \bar{\Omega}}\left(x_{0}\right)=\int_{0}^{t} D_{x} F\left(s, x_{i n}\left(s ; x_{0}\right)\right) D_{x} x_{i n}\left(s ; x_{0}\right) d s .
$$

Now, let $x_{0}, y_{0}$ be in $\bar{\Omega}$, then

$$
\begin{aligned}
& \left|\int_{0}^{t}\left(D_{x} F\left(s, x_{i n}\left(s ; x_{0}\right)\right) D_{x} x_{i n}\left(s ; x_{0}\right)-D_{x} F\left(s, x_{i n}\left(s ; y_{0}\right)\right) D_{x} x_{i n}\left(s ; y_{0}\right)\right)\right| d s \\
\leq & \int_{0}^{t}\left|D_{x} F\left(s, x_{i n}\left(s ; x_{0}\right)\right)-D_{x} F\left(s, x_{i n}\left(s ; y_{0}\right)\right)\right|\left|D_{x} x_{i n}\left(s ; x_{0}\right)\right| d s \\
+ & \int_{0}^{t}\left|D_{x} F\left(s, x_{i n}\left(s ; y_{0}\right)\right)\right|\left|D_{x} x_{i n}\left(s ; x_{0}\right)-D_{x} x_{i n}\left(s ; y_{0}\right)\right| d s .
\end{aligned}
$$


As was done in paragraph a) of this proof, multiplying this inequality by $\exp \left(-2 \int_{0}^{t} \rho(s) d s\right)$, and using (9) and the fact that $D_{x} x_{i n}\left(t ; x_{0}\right)$ is bounded in $\bar{\Omega}$, we have

$$
\begin{aligned}
\left\|D g_{\mid \bar{\Omega}}\left(x_{0}\right)-D g_{\mid \bar{\Omega}}\left(y_{0}\right)\right\|_{0}^{\prime} & \leq C^{\prime}\left\|x_{i n}\left((\cdot), x_{0}\right)-x_{i n}\left((\cdot), y_{0}\right)\right\|_{0}^{\alpha} \\
& +\frac{1}{2}\left\|D g_{\mid \bar{\Omega}}\left(x_{0}\right)-D g_{\mid \bar{\Omega}}\left(y_{0}\right)\right\|_{0}^{\prime},
\end{aligned}
$$

where $\|\cdot\|^{\prime}$ is the equivalent sup-norm defined in (12). The Lipschitz dependence of the solution of (6) with respect to the initial conditions implies that

$$
\left\|D g_{\mid \bar{\Omega}}\left(x_{0}\right)-D g_{\mid \bar{\Omega}}\left(y_{0}\right)\right\|_{0} \leq C^{\prime \prime}\left\|x_{0}-y_{0}\right\|_{0}^{\alpha} .
$$

So, $g_{\mid \bar{\Omega}}\left(x_{0}\right)$ belongs to the space $\mathcal{C}^{1, \alpha}(\bar{\Omega})$ and, therefore, the same will be true for $\Phi\left(x_{0}\right)=\lim _{t \rightarrow \infty} g_{\mid \bar{\Omega}}\left(x_{0}\right)$. Similar arguments can be reproduced to prove that $\Psi \in \mathcal{C}^{1, \alpha}(\bar{\Omega})$.

\section{Transport along the heat equation.}

This section is devoted to the proof of Theorem 1 stated in Section 1. With this aim, we are going to see that the hypotheses of Theorem 3 are satisfied with $F(t, x)=-\nabla u(t, x) / u(t, x)$, where $u$ is the solution of the heat equation with homogeneous Neumann boundary condition and initial value $f \in \mathcal{C}^{0, \alpha}(\bar{\Omega})$.

In order to prove that the $\mathcal{C}^{1,0}$-norm of $F(t, \cdot)$ is integrable in $(0, \infty)$ and that the condition (9) is fullfilled, we will need the second estimate of the following lemma. The first, which is a prior step of the proof of the second, is stated here because it will be used later in the proof of Theorem 2 .

Lemma 1 Let $v$ be a function of class $\mathcal{C}^{2, \beta}(\bar{\Omega})$ where $\Omega$ is a bounded domain of class $\mathcal{C}^{2, \alpha^{\prime}}$ with $0<\beta<\alpha^{\prime}$ and $0<r<R$ such that $r<v(x)<R$ for all $x \in \Omega$. Then, there exists a constant $C>0$, depending on $\Omega, r$ and $R$, such that

$$
\begin{aligned}
& \text { a) }\left\|\frac{1}{v}\right\|_{\mathcal{C}^{1, \beta}} \leq C\left(1+\|v-1\|_{\mathcal{C}^{2, \beta}}^{\frac{1+\beta}{2+\beta}}\right), \\
& \text { b) }\left\|\frac{\nabla v}{v}\right\|_{\mathcal{C}^{1, \beta}} \leq C\|v-1\|_{\mathcal{C}^{2, \beta}} .
\end{aligned}
$$

We remark that these statements remain true with $\|v-a\|_{\mathcal{C}^{2, \beta}}$, for any constant $a \in \mathbb{R}$, not necessarily $a=1$. But, they will used below only in the case that $a$ is the spatial average of $v$ that it will turn out to be 1 .

Proof: a) Using the following estimate for the $\mathcal{C}^{k, \beta}$-norm of a product of functions,

$$
\left\|v_{1} \cdots v_{j}\right\|_{\mathcal{C}^{k, \beta}} \leq \tilde{C} \sum_{i=1}^{j}\left(\left\|v_{i}\right\|_{\mathcal{C}^{k, \beta}} \prod_{m \neq i}\left\|v_{m}\right\|_{\mathcal{C}^{0,0}}\right)
$$


(see Theorem A.7 of Hörmander [14]) and the fact that $v(x)>r>0$ for all $x \in \Omega$, we have

$$
\begin{aligned}
\left\|\frac{1}{v}\right\|_{\mathcal{C}^{1, \beta}} & \leq \frac{1}{r}+\left\|\frac{\nabla v}{v^{2}}\right\|_{\mathcal{C}^{0, \beta}} \\
& \leq \frac{1}{r}+C_{1}\left(\frac{1}{r^{2}}\|\nabla v\|_{\mathcal{C}^{0, \beta}}+\frac{2}{r}\|\nabla v\|_{\mathcal{C}^{0,0}}\left\|\frac{1}{v}\right\|_{\mathcal{C}^{0, \beta}}\right) .
\end{aligned}
$$

Moreover, since

$$
\left\|\frac{1}{v}\right\|_{\mathcal{C}^{0, \beta}} \leq \frac{1}{r}+\sup _{x \neq y} \frac{|1 / v(x)-1 / v(y)|}{|x-y|^{\beta}} \leq \frac{1}{r}+\frac{1}{r^{2}}\|v-1\|_{\mathcal{C}^{0, \beta}},
$$

then

$$
\begin{aligned}
\left\|\frac{1}{v}\right\|_{\mathcal{C}^{1, \beta}} & \leq C_{2}\left(1+\|v-1\|_{\mathcal{C}^{1, \beta}}+\|\nabla v\|_{\mathcal{C}^{0,0}}+\|\nabla v\|_{\mathcal{C}^{0,0}}\|v-1\|_{\mathcal{C}^{0, \beta}}\right) \\
& \leq C_{3}\left(1+\|v-1\|_{\mathcal{C}^{1, \beta}}+\|v-1\|_{\mathcal{C}^{1,0}}\|v-1\|_{\mathcal{C}^{0, \beta}}\right) .
\end{aligned}
$$

Now, we estimate each term by using the interpolation inequality,

$$
\|v-1\|_{\mathcal{C}^{k, \gamma}} \leq C_{4}\|v-1\|_{\mathcal{C}^{2, \beta}}^{\frac{k+\gamma}{2+\beta}}\|v-1\|_{\mathcal{C}^{0,0}}^{1-\frac{k+\gamma}{2+\beta}}
$$

for $k=0,1$ and $\gamma=0, \beta$ (see Theorem A.5 of Hörmander [14]) and we obtain that

$$
\left\|\frac{1}{v}\right\|_{\mathcal{C}^{1, \beta}} \leq C_{5}\left(1+\|v-1\|_{\mathcal{C}^{2, \beta}}^{\frac{1+\beta}{2+\beta}}\|v-1\|_{\mathcal{C}^{0,0}}^{\frac{1}{2+\beta}}\right) .
$$

Finally, using that $v(x)<R$, we have the estimate (14).

$b$ ) For proving the second inequality, we use first the inequality (16), and after the estimate (14),

$$
\begin{aligned}
\left\|\frac{\nabla v}{v}\right\|_{\mathcal{C}^{1, \beta}} & \leq C_{6}\left(\|\nabla v\|_{\mathcal{C}^{1, \beta}} \frac{1}{r}+\|\nabla v\|_{\mathcal{C}^{0,0}}\left\|\frac{1}{v}\right\|_{\mathcal{C}^{1, \beta}}\right) \\
& \leq C_{7}\left(\|v-1\|_{\mathcal{C}^{2, \beta}}+\|v-1\|_{\mathcal{C}^{1,0}}\left(1+\|v-1\|_{\mathcal{C}^{2, \beta}}^{\frac{1+\beta}{2+\beta}}\right)\right) .
\end{aligned}
$$

Then, the estimate (15) follows by using the interpolation inequality (17) and that $v(x)<R$.

Proof of Theorem 1: a) First of all, we will show that $F(t, \cdot)=$ $-\nabla u(t, \cdot) / u(t, \cdot)$ is a continuous map from $(0, \infty)$ to $\mathcal{C}^{1,0}(\bar{\Omega})$. Under our regularity hypotheses for the boundary of $\Omega$, the initial value problem for the linear heat equation with homogeneous Neumann boundary conditions and initial value $u(0, x)=f(x) \in \mathcal{C}^{0, \alpha}(\bar{\Omega})$ defines a unique solution $u(t, x)$ continuous in $(t, x) \in[0, \infty) \times \bar{\Omega}$, and the solution curve $t \mapsto u(t, \cdot)$ is a continuous map from 
the open interval $(0, \infty)$ into the space $\mathcal{C}^{2,0}(\bar{\Omega})$. It is even locally Hölder continuous, though the Hölder constant can become unbounded as $t \rightarrow 0$ (see Belonosov [1], Solonnikov and Khachatryan [22], and also Mora's Theorem 2.3 [17]). Furthermore, by the maximum principle it follows that $u(t, x) \geq \inf _{\bar{\Omega}} f>0$.

The next step consists in obtaining estimates of $\|F(t, \cdot)\|_{\mathcal{C}^{1, \beta}}$ for $0 \leq \beta<\alpha^{\prime}$. For $\beta=0$, these estimates will imply that $\|F(t, \cdot)\|_{\mathcal{C}^{1,0}} \in L^{1}(0, \infty)$, and for a suitable choice of $\beta$, they will imply inequality (9) in the proof of paragraph $b$ ).

For $0<t<1$, using the inequalilty (15) of Lemma 1 and the following crucial estimate for the solution to the heat equation

$$
\|u(t, \cdot)\|_{\mathcal{C}^{2, \beta}} \leq \frac{C}{t^{1-(\alpha-\beta) / 2}}\|f\|_{\mathcal{C}^{0, \alpha}}, \quad \text { for all } 0<\alpha<1,
$$

(see Belonosov [1] and Mora [17]) we obtain that

$$
\|F(t, \cdot)\|_{\mathcal{C}^{1, \beta}} \leq C\|u(t, \cdot)-1\|_{\mathcal{C}^{2, \beta}} \leq \frac{C}{t^{1-(\alpha-\beta) / 2}}\|f-1\|_{\mathcal{C}^{0, \alpha}} .
$$

In particular, taking $\beta=0$ in (18) and since in our case $0<\alpha<1$, we deduce that $\|F(t, \cdot)\|_{\mathcal{C}^{1,0}}$ is integrable near $t=0$.

Moreover, one of Mora's results [17] shows that the linear heat equation with homogeneous Neumann boundary conditions defines an analytic semigroup in the space $\mathcal{C}^{0,0}(\bar{\Omega})$. So, for all $t>0, u(t, \cdot)$ belongs to all the spaces $D^{\gamma}, \gamma>0$, of domains of the fractional powers of the infinitesimal generator of the semigroup. In the same paper, it is shown that these spaces $D^{\gamma}$ are continuously embedded into $\mathcal{C}^{2, \beta}(\bar{\Omega})$ for all $\gamma>1+\beta / 2$ (see Theorem 2.5 of [17]). Hence, there exists a constant $C_{\gamma}>0$ such that

$$
\|u(t, \cdot)-1\|_{\mathcal{C}^{2, \beta}} \leq C_{\gamma}\|u(t, \cdot)-1\|_{D^{\gamma}} .
$$

Moreover, since the function $u(t, \cdot)-1$ is orthogonal to the constant eigenfunction of the Laplacian with Neumann boundary conditions (i.e. 1 is the spatial average of $u$ )

$$
\int_{\Omega}(u(t, x)-1) d x=\int_{\Omega} u(0, x) d x-|\Omega|=\int_{\Omega} f(x) d x-|\Omega|=0,
$$

and then

$$
\|u(t, \cdot)-1\|_{\mathcal{C}^{2, \beta}} \leq C_{\gamma}\|u(t, \cdot)-1\|_{D^{\gamma}} \leq C e^{-\delta(t-1)}\|u(1, \cdot)-1\|_{D^{\gamma}},
$$

for $t \geq 1$ and where $\delta$ is a positive value lower than the second eigenvalue of the Laplace operator with homogeneous Neumann boundary conditions (the proof of this inequality can be found in Theorem 1.4.3 of Henry's book [15], in the setting of semigroup theory). Then, joining the inequalities (15), (19) and (20) we obtain that, for $1 \leq t<\infty$,

$$
\|F(t, \cdot)\|_{\mathcal{C}^{1, \beta}} \leq C e^{-\delta t} .
$$


In particular, taking $\beta=0$, we show that $\|F(t, \cdot)\|_{\mathcal{C}^{1,0}}$ is integrable near $t=\infty$. Therefore, paragraph $a$ ) follows from the same paragraph of Theorem 3.

b) In order to prove that the map $\Phi$ is $\mathcal{C}^{1, \alpha}$, we will see that the inequality (9) is fulfilled. First of all, we observe that (9) with an exponent $\beta<\alpha$ instead of $\alpha$ is a direct consequence of (18) and (21), since these inequalities would prove that $\|F(t, \cdot)\|_{\mathcal{C}^{1, \beta}} \in L^{1}(0, \infty)$. But the limit case, $\beta=\alpha$, is less simple, and we use for its proof an argument taken from Rivière and Ye [19].

Let us write $h=\|x-y\|_{0}$ and let us restrict without loss of the case $h<1$. By using (18) with $\beta=0$, (18) for some $\alpha<\beta<\alpha^{\prime}$, and (21) with $\beta=\alpha$, we have

$$
\begin{aligned}
& \int_{0}^{\infty}\left|D_{x} F(s, x(s))-D_{x} F(s, y(s))\right| d s \\
= & \left(\int_{0}^{h^{2}}+\int_{h^{2}}^{1}+\int_{1}^{\infty}\right)\left|D_{x} F(s, x(s))-D_{x} F(s, y(s))\right| d s \\
\leq & \int_{0}^{h^{2}} 2\|F(s, \cdot)\|_{\mathcal{C}^{1,0}} d s+\int_{h^{2}}^{1}\|F(s, \cdot)\|_{\mathcal{C}^{1, \beta}} h^{\beta} d s+\int_{1}^{\infty}\|F(s, \cdot)\|_{\mathcal{C}^{1, \alpha}} h^{\alpha} d s \\
\leq & \int_{0}^{h^{2}} \frac{C}{s^{1-\alpha / 2}} d s+\int_{h^{2}}^{1} \frac{C h^{\beta}}{s^{1-(\alpha-\beta) / 2}} d s+\int_{1}^{\infty} C e^{-\delta s} h^{\alpha} d s \\
\leq & C^{\prime} h^{\alpha} .
\end{aligned}
$$

Finally, let us calculate $\int_{0}^{\infty} \operatorname{div} F(s, x(s)) d s$,

$$
\begin{aligned}
\operatorname{div}\left(-\frac{\nabla u}{u}\right) & =-\frac{\Delta u}{u}+\frac{\nabla u}{u} \cdot \frac{\nabla u}{u}=-\frac{1}{u}\left(\frac{\partial u}{\partial t}+\nabla u \cdot x^{\prime}(t)\right) \\
& =-\frac{1}{u} \frac{D}{D t}[u(t, x(t))]=-\frac{D}{D t}[\ln (u(t, x(t)))]
\end{aligned}
$$

and so,

$$
\int_{t_{1}}^{t_{2}} \operatorname{div} F(s, x(s)) d s=\ln \frac{u\left(t_{1}, x\left(t_{1}\right)\right)}{u\left(t_{2}, x\left(t_{2}\right)\right)} .
$$

As one sees from $(20), u(t, \cdot)-1$ tends uniformly to 0 as $t \rightarrow \infty$. And we also know that $u(t, x(t)) \rightarrow f\left(x_{0}\right)$ as $t \rightarrow 0$. So, the limit of this integral when $t_{1} \rightarrow 0$ and $t_{2} \rightarrow \infty$ is $\ln \left(f\left(x_{0}\right)\right)$, and therefore using (8), we obtain that $\operatorname{det} D \Phi\left(x_{0}\right)=f\left(x_{0}\right)$, for all $x_{0} \in \bar{\Omega}$.

\section{The viscous modification}

In this section we will prove Theorem 2 stated in Section 1. With this aim, we will see that the hypotheses of Theorem 3 are satisfied with $F(t, x)=$ $-(\nabla u(t, x)-V(t, x)) / u(t, x)$, where $u$ is the solution of the heat equation with 
homogeneous Neumann boundary conditions and initial condition $f$, and for each $t>0, V(t, x)$ is the weak solution $W$ of the Stokes system

$$
\left\{\begin{aligned}
\Delta W(x) & =\nabla p(x), & & x \in \Omega, \\
\operatorname{div} W(x) & =0, & & x \in \Omega, \\
W(x) & =W^{*}(x), & & x \in \partial \Omega,
\end{aligned}\right.
$$

when $W^{*}(x)=\nabla u(t, x)$

First of all, we introduce the auxiliar spaces

$$
\begin{aligned}
\mathcal{C}_{\sigma}^{k, \beta}(\partial \Omega) & =\left\{W^{*} \in \mathcal{C}^{k, \beta}(\partial \Omega): \int_{\partial \Omega} W^{*} \cdot \nu=0\right\}, \\
H_{0, \sigma}^{1}(\Omega) & =\left\{\phi \in H^{1}(\Omega): \operatorname{div} \phi=0 \text { in } \Omega, \phi=0 \text { at } \partial \Omega\right\} .
\end{aligned}
$$

Then, given $W^{*} \in \mathcal{C}_{\sigma}^{1,0}(\partial \Omega)$, a vector function $W \in \mathcal{C}^{1,0}(\bar{\Omega})$ will be called a weak solution of the Stokes system (22) if $\operatorname{div} W=0$ in $\Omega, W=W^{*}$ at $\partial \Omega$ and $\int_{\Omega} \nabla W \cdot \nabla \phi=0$, for all $\phi \in H_{0, \sigma}^{1}(\Omega)$.

We observe that this definition is not the most general notion of weak solution, but it will be enough for our purposes.

Next, we present a lemma about the existence and regularity of this class of solutions.

Lemma 2 Let $\Omega \subset \mathbb{R}^{n}$ be a bounded domain of class $\mathcal{C}^{2, \alpha^{\prime}}$ and $\beta$ such that $0<\beta<\alpha^{\prime}<1$. For all $W^{*} \in \mathcal{C}_{\sigma}^{1, \beta}(\partial \Omega)$, there exists a unique weak solution $W$ of the Stokes problem (22) such that $W \in \mathcal{C}^{1, \beta}(\bar{\Omega})$ and satisfies:

$$
\begin{aligned}
\|W\|_{\mathcal{C}^{0,0}} & \leq C\left\|W^{*}\right\|_{\mathcal{C}_{\sigma}^{0,0}}, \\
\|W\|_{\mathcal{C}^{1, \beta}} & \leq C\left\|W^{*}\right\|_{\mathcal{C}_{\sigma}^{1, \beta}}
\end{aligned}
$$

Proof: Let us consider first $W^{*} \in \mathcal{C}_{\sigma}^{2, \beta}(\partial \Omega)$. In this case, it is known the existence and uniqueness of a classical solution $W \in \mathcal{C}^{2, \beta}(\bar{\Omega})$ that it satisfies

$$
\|W\|_{\mathcal{C}^{2, \beta}} \leq C_{2, \beta}\left\|W^{*}\right\|_{\mathcal{C}_{\sigma}^{2, \beta}},
$$

for some $C_{2, \beta}>0$ (see Solonnikov [21] and Theorem 7.2 of Galdi [10]). Moreover, from the maximum modulus principle for the Stokes system, we have also

$$
\|W\|_{\mathcal{C}^{0,0}} \leq C_{0,0}\left\|W^{*}\right\|_{\mathcal{C}_{\sigma}^{0,0}},
$$

for some $C_{0,0}>0$ (see Galdi and Varnhorn [11], or also, the notes for Section IV.6 of Galdi [10]).

Now, from these two inequalities, and using that $\mathcal{C}_{\sigma}^{2, \beta}(\partial \Omega)$ is dense in $\mathcal{C}_{\sigma}^{0,0}(\partial \Omega)$, we have that the solution map of the Stokes system (22)

$$
\begin{aligned}
T_{2, \beta}: \mathcal{C}_{\sigma}^{2, \beta}(\partial \Omega) & \rightarrow \mathcal{C}^{2, \beta}(\bar{\Omega}) \\
W^{*} & \rightarrow W
\end{aligned}
$$


extends to a bounded linear map $T_{0,0}: \mathcal{C}_{\sigma}^{0,0}(\partial \Omega) \rightarrow \mathcal{C}^{0,0}(\bar{\Omega})$.

According to Triebel (see Theorem 2.4.2 of [23]), for $0<\beta<1$, we consider $\mathcal{C}^{1, \beta}(\bar{\Omega})$ as the interpolation space $\left(\mathcal{C}^{0,0}(\bar{\Omega}), \mathcal{C}^{2, \beta}(\bar{\Omega})\right)_{\theta, \infty}$ with $\theta=(1+\beta) /(2+\beta)$. So, the map $T_{0,0}$ restricts to a bounded linear map $T_{1, \beta}: \mathcal{C}_{\sigma}^{1, \beta}(\partial \Omega) \rightarrow \mathcal{C}^{1, \beta}(\bar{\Omega})$ such that, if $W^{*} \in \mathcal{C}_{\sigma}^{2, \beta}(\partial \Omega)$, then $T_{2, \beta} W^{*}=T_{1, \beta} W^{*}$ and

$$
\|W\|_{\mathcal{C}^{1, \beta}} \leq C_{1, \beta}\left\|W^{*}\right\|_{\mathcal{C}_{\sigma}^{1, \beta}} .
$$

Note that Triebel [23] works with domains of $\mathcal{C}^{\infty}$ class, but the same arguments work in our case since $\mathcal{C}^{0,0}(\bar{\Omega})$ and $\mathcal{C}^{2, \beta}(\bar{\Omega})$ can be taken as factor spaces of $\mathcal{C}^{0,0}\left(\mathbb{R}^{n}\right)$ and $\mathcal{C}^{2, \beta}\left(\mathbb{R}^{n}\right)$, respectively, with a common projection.

Let us consider now $W^{*} \in \mathcal{C}_{\sigma}^{1, \beta}(\partial \Omega)$. We want to see that $T_{1, \beta} W^{*}$ is a weak solution of (22). It is known that there exists a sequence $\left(W_{n}^{*}\right) \in \mathcal{C}_{\sigma}^{2, \beta}(\partial \Omega)$ such that $\left\|W_{n}^{*}\right\|_{\mathcal{C}_{\sigma}^{1, \beta}}$ is bounded independently of $n$ and converges to $W^{*}$ in $\mathcal{C}_{\sigma}^{1, \gamma}(\partial \Omega)$ for each $0 \leq \gamma<\beta$ (see Section 2 of Chapter 7 of Gilbarg and Trudinger [12]). For our previous arguments, $T_{2, \beta} W_{n}^{*}$ is bounded in $\mathcal{C}^{1, \beta}(\bar{\Omega})$ and has a subsequence converging in $\mathcal{C}^{1,0}(\bar{\Omega})$ to some $W \in \mathcal{C}^{1, \beta}(\bar{\Omega})$. Thus, since $T_{2, \beta} W^{*}$ is the classical solution, $W$ is a weak solution of (22). Furthermore, it is easy to see that it is the only weak solution and, since $W=T_{0,0} W^{*}=T_{1, \beta} W^{*}$, this weak solution satisfies inequalities (24) and (23).

Proof of Theorem 2: a) First of all, we consider

$$
F(t, \cdot)=-\frac{\nabla u(t, \cdot)-V(t, \cdot)}{u(t, \cdot)}
$$

as a map from $(0, \infty)$ to $\mathcal{C}^{1,0}(\bar{\Omega})$. By using Mora's characterization [17] of the domains $D^{\gamma}$ of the fractional powers of the generator of the heat semigroup, as we did in the proof of Theorem 1 , we have that $\nabla u(t, \cdot):(0, \infty) \rightarrow \mathcal{C}^{1, \beta}(\bar{\Omega})$ is continuous for $0 \leq \beta<\alpha^{\prime}$. Moreover, because of inequality (24), $V$ depends continuously on $\nabla u$ in the $\mathcal{C}^{1, \beta}$-norms. Therefore, similar to the proof of Theorem 1, we have that $F:(0, \infty) \rightarrow \mathcal{C}^{1, \beta}\left(\bar{\Omega}, \mathbb{R}^{n}\right) \subset \mathcal{C}^{1,0}(\bar{\Omega})$ is continuous.

Next we will prove that $\|F\|_{\mathcal{C}^{1,0}}$ is integrable in $(0, \infty)$. So, from inequality (16), the first estimate of Lemma 1, the maximum principle for $u(t, x)$, Lemma 2 and the interpolation inequality (17), we have for $0 \leq \beta<\alpha^{\prime}$,

$$
\begin{aligned}
\left\|\frac{V(t, \cdot)}{u(t, \cdot)}\right\|_{\mathcal{C}^{1, \beta}} & \leq C_{1}\left(\|V(t, \cdot)\|_{\mathcal{C}^{1, \beta}} \frac{1}{r}+\|V(t, \cdot)\|_{\mathcal{C}^{0,0}}\left\|\frac{1}{u(t, \cdot)}\right\|_{\mathcal{C}^{1, \beta}}\right) \\
& \leq C_{2}\left(\|\nabla u(t, \cdot)\|_{\mathcal{C}^{1, \beta}}+\|\nabla u(t, \cdot)\|_{\mathcal{C}^{0,0}}\left(1+\|u(t, \cdot)-1\|_{\mathcal{C}^{2, \beta}}^{\frac{1+\beta}{2+\beta}}\right)\right) \\
& \leq C_{3}\|u(t, \cdot)-1\|_{\mathcal{C}^{2, \beta}}
\end{aligned}
$$

where $0<r<\inf _{\bar{\Omega}} f$. Now, taking into account the estimates (18) and (21), we obtain that

$$
\begin{aligned}
\|F\|_{\mathcal{C}^{1, \beta}} & \leq \frac{C}{t^{1-(\alpha-\beta) / 2}}, \quad 0<t \leq 1, \quad \text { for all } 0 \leq \beta<\alpha^{\prime} \\
\|F\|_{\mathcal{C}^{1, \alpha}} & \leq C e^{-\delta t}, \quad t \geq 1
\end{aligned}
$$


So, in particular $\|F(t, \cdot)\|_{\mathcal{C}^{1,0}} \in L^{1}(0, \infty)$.

Finally, the inequality

$$
\int_{0}^{\infty}\left|D_{x} F(t, x(t))-D_{x} F(t, y(t))\right| d t \leq C\|x-y\|_{0}^{\alpha}
$$

is proved in the same way as in Theorem 1, but using estimates (26) and (27) instead of estimates (18) and (21).

b) To compute $\operatorname{det}(D \Phi)(x)$, we calculate $\int_{0}^{\infty} \operatorname{div} F(t, x(t)) d t$ along a solution $x(t)$ of $(5)$,

$$
\begin{aligned}
\operatorname{div} F & =\operatorname{div}\left(-\frac{\nabla u-V}{u}\right)=-\frac{\Delta u}{u}+\frac{\nabla u}{u} \cdot \frac{\nabla u-V}{u} \\
& =-\frac{1}{u}\left(\frac{\partial u}{\partial t}+\nabla u \cdot x^{\prime}(t)\right) \\
& =-\frac{1}{u} \frac{D}{D t}(u(t, x(t)))=-\frac{D}{D t}(\ln (u(t, x(t)))),
\end{aligned}
$$

and the rest of the calculation is the same as in the proof of Theorem 1.

Lastly, one also observes that for $x \in \partial \Omega$ one has $\Phi(x)=x$, since $F(t, x)=0$ for all $t>0$.

\section{References}

[1] V. S. Belonosov, "Estimates of solutions of parabolic systems in weighted Hölder classes and some of their applications", Mat. Sb., 110, (1979), 163188. English Trans: Math. USSR Sb. 38, (1979), 151-173.

[2] Y. Brenier, "Polar factorization and monotone rearrangement of vectorvalued functions", Comm. Pure Appl. Math., 44, (1991), 375-417

[3] D. Burago, B. Kleiner, "Separated Nets in Euclidean Space and Jacobians of Bilipschitz Maps", GAFA, Geom. funct. anal. 2, (1998), 273-282.

[4] L. Caffarelli, "Some regularity properties of solutions of Monge-Ampère equation”,, Comm. Pure Appl. Math. 44, (1991), 965-969.

[5] S.N. Chow, J. K. Hale, Methods of Bifurcation Theory, Sringer-Verlag, 1982.

[6] B. Dacorogna, Direct Methods in the Calculus of Variations, SpringerVerlag, 1989.

[7] B. Dacorogna, J. Moser, "On a partial differential equation involving the Jacobian determinant", Ann. Inst. Henri Poincaré, 49, 1, (1990), 1-26.

[8] D. Dorling, Area Cartograms: Their Use and Creation, Concepts and Techniques of Modern Geography, 59, Institute of British Geographers, 1996. 
[9] L. C. Evans, W. Gangbo, Diferential Equations Methods for the MongeKantorovich Problem, Memoirs of AMS, 137, 1999.

[10] G. P. Galdi, An Introduction to the Mathematical Theory of the NavierStokes Equations, Vol I, Springer-Verlag, 1994.

[11] G. P. Galdi, W. Varnhorn, "The Maximum Modulus Theorem for the Stokes System", (to be published).

[12] D. Gilbarg, N. S. Trudinger, Elliptic Partial Differential Equations, (Second Edition, Revised Third Printing), Springer-Verlag, 1998.

[13] P. Hartman, Ordinary Differential Equations, J. Wiley, 1964.

[14] L. Hörmander, "The boundary problems of Physical Geodesy", Arch. Ration. Mech. Anal. 120, (1976), 1-52.

[15] D. Henry, Geometric theory of semilinear parabolic equations, Lecture Notes in Math., 840, Springer-Verlag, 1981.

[16] C. McMullen, "Lipschitz Maps and Nets in Euclidean Space", GAFA, Geom. funct. anal. 2, (1998), 304-314.

[17] X. Mora, "Semilinear parabolic problems define semiflows on $C^{k}$ spaces", Trans. Amer. Math. Soc. 278, (1983), 21-55.

[18] J. Moser, "On the volume elements on a manifold", Trans. Amer. Math. Soc. 120, (1965), 286-294.

[19] T. Rivière and D. Ye, "Resolutions of the prescribed volume form equation", Nonlinear Differential Equations Appl. 3, (1996), 323-369.

[20] G. Russo, "Deterministic diffusion of particles", Comm. Pure Appl. Math., XLIII, (1990), 697-733.

[21] V. A. Solonnikov, "General Boundary Value Problems for DouglisNirenberg Elliptic Systems II, Trudy. Mat. Inst. Steklov, 92 (1966), 233-297. English Trans: Proc. Steklov Inst. Math., 92, (1966), 212-272.

[22] V. A. Solonnikov, A. G. Khachatryan, "Estimates for solutions of parabolic initial-boundary value problems in weighted Hölder norms", Trudy Mat. Inst. Steklov, 147, (1980), 147-155. English Trans: Proc. Steklov Math. Inst., 147, (1980), 153-162.

[23] H. Triebel, Interpolation theory, Function Spaces, Differential Operators, North-Holland, 1978. 
1 Departament de Matemàtica Aplicada 2. Universitat Politècnica de Catalunya. C. Pau Gargallo, 5. 08028-Barcelona, Spain. e-mail: avinyo@ma2.upc.es.

2 Departament de Matemàtica Aplicada 1. Universitat Politècnica de Catalunya. Av. Diagonal, 647. 08028-Barcelona, Spain. e-mail: jsola@ma1.upc.es, valencia@ma1.upc.es. 